\title{
Birefringence swap at the transition to hyperbolic dispersion in metamaterials
}

\author{
L. M. Custodio, ${ }^{1}$ C. T. Sousa, ${ }^{1}$ J. Ventura, ${ }^{1}$ J. M. Teixeira, ${ }^{1}$ P. V. S. Marques,${ }^{2,3}$ and J. P. Araujo ${ }^{1,2, *}$ \\ ${ }^{1}$ IFIMUP/IN-Instituto de Nanociência e Nanotecnologia, R. Campo Alegre 4169-007 Porto, Portugal \\ ${ }^{2}$ Universidade do Porto-DFA Faculdade de Ciências, R. Campo Alegre, 4169-007 Porto, Portugal \\ ${ }^{3}$ INESC Porto-UOSE, R. Campo Alegre 4169-007 Porto, Portugal
}

(Received 4 November 2011; revised manuscript received 9 January 2012; published 5 April 2012)

\begin{abstract}
The Bruggeman effective medium is used to study the transition to hyperbolic dispersion of visible light in thin-film metal-dielectric composite metamaterial of varying mixing proportion. This transition is experimentally demonstrated by the detection of the swap between the refracted birefringence components in fabricated composites of silver nanowires embedded in anodic aluminium oxide. Three refraction regimes are observed in a single composite using excitation radiation on both sides of the transition.
\end{abstract}

DOI: 10.1103/PhysRevB.85.165408

PACS number(s): 81.05.Xj, 78.20.Fm, 78.67.Pt, 81.07.Gf

\section{INTRODUCTION}

Metamaterials are artificial structures designed to offer specific electromagnetic (macroscopic) responses usually not found in nature. Formally, the first metamaterial was conceived to demonstrate negative refraction as the result of an effective negative refractive index. ${ }^{1}$ The possibility of such a refractive index proposed three decades before raised several extraordinary optical properties such as negative refraction, amplification of evanescent fields, reversed Doppler and Cherenkov effects, special Brewster and critical angles. ${ }^{2}$ However, it was the metamaterial experimental solution (meta-atoms) and the perspective of practical applications ${ }^{3}$ that fueled a strong research on these and other types of optical media. An almost immediate consequence of such a trend was the first formal study on the indefinite media. ${ }^{4}$ The term was applied to all anisotropic media in which the principal components of the diagonalized electromagnetic permittivity and permeability tensors $\left(\epsilon_{i}\right.$ and $\mu_{i}$ with $\left.i=x, y, z\right)$ do not all have the same sign. One of the most relevant attributes of the indefinite media is the hyperbolic-shaped dispersion, which can cause negative refraction. As a direct example, for a $p$-polarization incidence in a lossless indefinite media with interface normal to the $z$ direction, one can have two media types satisfying either one of the conditions ${ }^{4}: \mu_{y} \epsilon_{x}<0$ and $\epsilon_{x} / \epsilon_{z}<0$, or $\mu_{y} \epsilon_{x}>0$ and $\epsilon_{x} / \epsilon_{z}<0$; and for each of these, one can have two subtypes that offer positive or negative refraction. ${ }^{5}$ From this, one can immediately understand that such conditions can also be satisfied with nonmagnetic indefinite permittivity (IP) media. A practical IP can be obtained with different types of metal-dielectric nanostructures, ${ }^{6}$ and the research on this field rapidly began to output extraordinary applications. ${ }^{7}$

In this work, we have designed, produced, and studied the IP features of thin-film nanowire composites consisting of vertically aligned high-aspect-ratio silver nanowires embedded in anodic aluminium oxide (Ag/AAO). Nanowire composites are currently being used to develop a variety of remarkable applications such as hyperlenses, ${ }^{8}$ cloaking and photonic devices, ${ }^{9,10}$ nanosensors, ${ }^{11}$ and other plasmonic devices. Following the results of a local effective-medium model, ${ }^{12}$ samples of the Ag/AAO nanowire composite were prepared with a range of different metal fill-ratios using a versatile template-assisted method. ${ }^{13}$ The samples were then submitted to optical microscope observations of the visible light exiting birefringence components, and the transition to hyperbolic dispersion was demonstrated at the fill-ratio where a swap between the components occurs. Additionally, three characteristic refraction regimes were demonstrated in a single composite, using near-infrared and visible light (IP, and near IP conditions). These regimes are equivalent to having a refractive index of $n>0$ (regular refraction), $0<n<1$ (low refraction), and $n<0$ (negative refraction). Finally, a comparison was established between the modeling and experimental results and with the experimental results of other recent works on nanowire composites.

\section{EFFECTIVE-MEDIUM MODEL}

The Bruggeman effective medium is suitable for (nonmagnetic) two-phase composites with high-aspect-ratio inclusions. ${ }^{14}$ The model derives from an approximation of the Maxwell-Garnett theory, ${ }^{15}$ and the used quasistatic limit approach is applicable when the inclusions size and spacing are small compared to the used radiation wavelength. The approximation leads to the solution (see Appendix A)

$$
\begin{aligned}
\epsilon(\eta, f) & =\left(\Delta \pm \sqrt{\Delta^{2}+4 \eta \epsilon_{i} \epsilon_{m}}\right) / 2 \eta, \\
\Delta & =f(1+\eta)\left(\epsilon_{m}-\epsilon_{i}\right)+\eta \epsilon_{i}-\epsilon_{m},
\end{aligned}
$$

where the sign \pm is chosen to satisfy $\operatorname{Im}(\epsilon)>0$. In the expression, $\epsilon$ is the effective-medium permittivity, $\epsilon_{i}$ and $\epsilon_{m}$ refer, respectively, to the local response of the inclusions and matrix phases (metal and dielectric), $\eta$ and $f$ are the inclusions screening factor and fill-ratio. For sphere-type inclusions $\eta=$ $2,{ }^{15}$ whereas for long-cylinder-type inclusions $\eta_{t}=1$ and $\eta_{l}=$ $\infty,{ }^{16}$ which results, respectively, in the transverse $\left(\epsilon_{t}\right)$ and longitudinal $\left(\epsilon_{l}\right)$ permittivity components of uniaxial media with optical axis in the transverse direction (to the media interface). Consequently, if the intrinsic dispersion of each constituent is explicitly taken into account, the effective permittivities can be calculated as a function of the relevant parameters $f$ and $\lambda$. These solutions can then be used to map an expanded representation of the parameter $\alpha(f, \lambda)=n_{l}^{2} / n_{t}^{2}$, where $n_{j=l, t}^{2}(f, \lambda)=$ $\operatorname{Re}\left(\epsilon_{j}\right)\left\{1+\sqrt{1+\left[\operatorname{Im}\left(\epsilon_{j}\right) / \operatorname{Re}\left(\epsilon_{j}\right)\right]^{2}}\right\} / 2$ are the longitudinal and transverse squared refractive index (the dependencies on $\lambda$ and $f$ are defined implicit henceforth). The latter relation is a general form of the expression that relates the complex dielectric response with the refractive index and extinction 

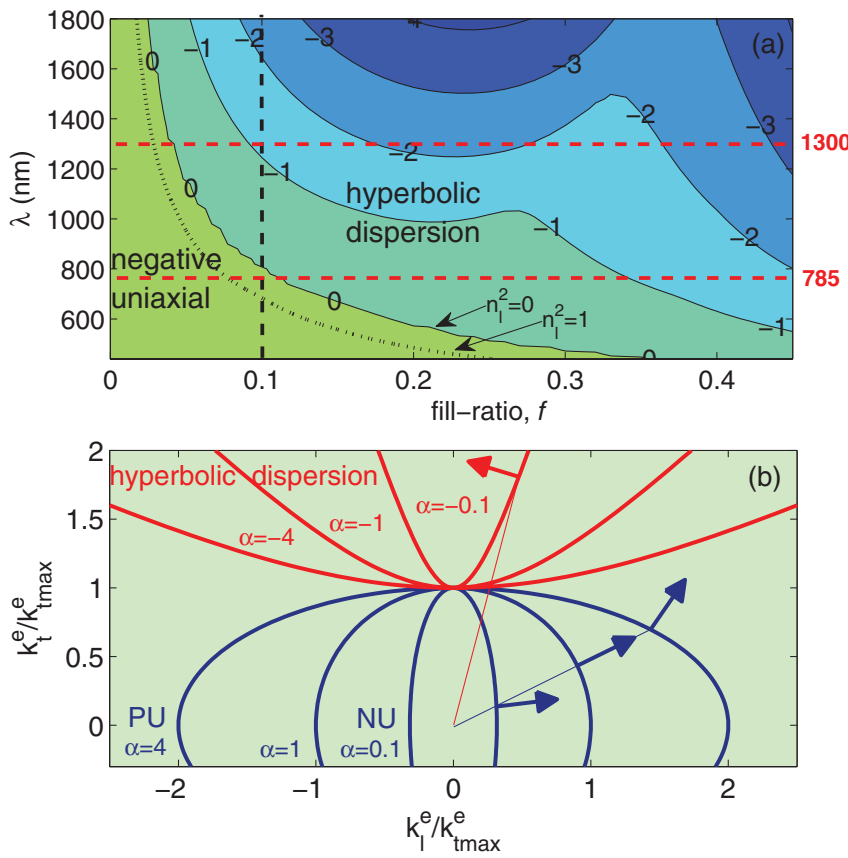

FIG. 1. (Color online) (a) $\alpha$-map representation (dashed lines indicate the tested regions); (b) extraordinary wave characteristic equifrequency curves (normalized by the transverse wave vector $k_{t \max }^{e}$ ) of the $\alpha$ values present in (a).

coefficient $\tilde{\epsilon}=(n-i k)^{2} \cdot{ }^{17}$ In the following normal surface analysis (quadric surfaces) and optical refraction studies, the squared refractive index is considered (instead of the dielectric constant) since it provides more immediate and practical information, and almost all the necessary response information is contained in the defined $\alpha$ coefficient. In the present case of nearly transparent media, the extinction coefficient results negligible and the quadric surfaces can then be described using $\left(x^{2}+y^{2}\right) / n_{t}^{2}+z^{2} / n_{l}^{2}=1$, with $n_{l, t}^{2}$ calculated as above.

Figure 1(a) illustrates the mapped results ( $\alpha$-map) comprising all possible IP conditions in the visible spectral region. Represented in Fig. 1(b) are the $\alpha$-map characteristic equifrequency curves of the refracted wave vectors resulting from a $p$-polarization incidence (extraordinary wave component) $k^{e}$. The representation is normalized by the transverse wave vector at maximum projection angle $k_{t \text { max }}^{e}$, and from this we can clearly understand that for $\alpha>1$ conditions, one has the regular elliptic-type dispersion of a positive uniaxial (PU). For $\alpha=1$, the isotropic case arises, and for $0<\alpha<1$, one has a negative uniaxial (NU). The dispersion of $\alpha$ results essentially from $n_{l}^{2}$, and if this component drops below unity [thin dotted line in Fig. 1(a)] the refraction angle of the extraordinary wave becomes higher than the incident angle (low refraction). Finally, for $\alpha<0$, one has the hyperbolic-type dispersion (HD), as a consequence of an indefinite permittivity.

On each equifrequency curve, one can also identify the walkoff angle $\delta$ of the power-flux vector $S^{e}$ (thick arrows), with regard to the represented $k^{e}$ (thin arrows). The direction of $S^{e}$ can be generally associated to the group velocity [ $v_{g}=$ $\left.\nabla_{k} \omega(k)\right]$, which results normal to the equifrequency curve. The ordinary wave, resulting from an $s$-polarization incidence, only depends on the $n_{t}^{2}$ component which has a negligible dispersion (not shown), and for this reason, both $S^{o}$ and $k^{o}$ closely follow the same direction of $k^{e}$. If we examine the $S^{e}$ vectors due to positives $\alpha$ (dark arrows), in the PU case it results in a counterclockwise angle $\delta$. However, if from varying the $(\lambda, f)$ parameters $\alpha$ decreases below unity (NU case), we have a birefringence components swap ( $S^{e}$ crosses with $k^{e}$ and $S^{o}$ ), and $\delta$ changes to clockwise. If $\alpha$ continues to decrease further toward zero, then $S^{e}$ will gradually approach the longitudinal component direction (refraction as with incidence at critical angle). At the particular condition where $\alpha$ crosses zero to become negative (transition to HD where a strong longitudinal plasmonic resonance arises), it results from the local model that the elliptic dispersion flattens and changes into hyperbolic. Due to this, the $S^{e}$ solution immediately jumps to the opposite direction of the longitudinal axis, crossing again with $k^{e}$ and $S^{o}$, and offering negative refraction. Then, as the negative $\alpha$ continues to decrease further, $S^{e}$ will gradually approach the transverse direction. The above-described dispersion can be quantified by applying the model results in the Snell law equation, which is coupled with a second known equation for anisotropic media (see Appendix B)

$$
1 / n_{e}^{2}\left(\theta_{t r}\right)=\cos ^{2} \theta_{t r} / n_{t}^{2}+\sin ^{2} \theta_{t r} / n_{l}^{2},
$$

where $n_{t}=n_{o}$ and $n_{l}=n_{e}$. Note that in this equation, the extraordinary wave refractive index $\left(n_{e}\right)$ depends on the angle of $k^{e}$ with respect to the interface normal $\left(\theta_{t r}\right)$. By solving the coupled equations, one obtains

$$
\begin{aligned}
\sin ^{2} \theta_{t r} & =\left(n_{l}^{2} / n_{i n c}^{2} \sin ^{2} \theta_{i n c}+1-n_{t}^{2} / n_{l}^{2}\right)^{-1}, \\
\tan \delta & =\frac{n_{i n c}^{2} \sin ^{2} \theta_{i n c} \sin 2 \theta_{t r}}{2 \sin ^{2} \theta_{t r}}\left(\frac{1}{n_{l}^{2}}-\frac{1}{n_{t}^{2}}\right) .
\end{aligned}
$$

For a non-normal incidence, one can use these solutions to determine the extraordinary wave deviation at the output of the sample, with respect to a normal incidence. To evaluate the birefringence separation, one also needs to find the ordinary wave refraction angle using the regular Snell law.

\section{SAMPLE PREPARATION}

The expanded representation in Fig. 1(a) highlights all the engineering possibilities of the nanowire composite and immediately motivates an experimental study on the transition to $\mathrm{HD}$ by varying the fill-ratio. Following this objective, AAO templates were synthesized using a two-step anodization method $\left(40 \mathrm{~V}\right.$ under oxalic acid at $\left.2{ }^{\circ} \mathrm{C}\right) .{ }^{18}$ This results in a nanoporous structure, self-assembled in a vertical uniform parallel arranged array [Fig. 2(a)], offering a natural 10\% porosity (average pore diameter of $d \sim 35 \mathrm{~nm}$ spaced by $D \sim 70 \mathrm{~nm}) .{ }^{19}$ To produce the Ag/AAO composite of different fill-ratios, the nanopores were widened using a controlled chemical etch process, and the silver nanowires were then grown inside using a pulsed electrodeposition method. ${ }^{20}$ The metal fill-ratio was determined using $f=c \cdot p$, where $c$ is the proportion of filled pores and $p=(\pi / \sqrt{12})(d / D)^{2}$ is the widened template porosity, both evaluated by scanning electron microscopy (SEM). Samples were produced with an average thickness of $5 \mu \mathrm{m}$ and, after electrodeposition, the top surface was ion milled until the variation of filled pores with depth was not significant. An opaque silver layer 

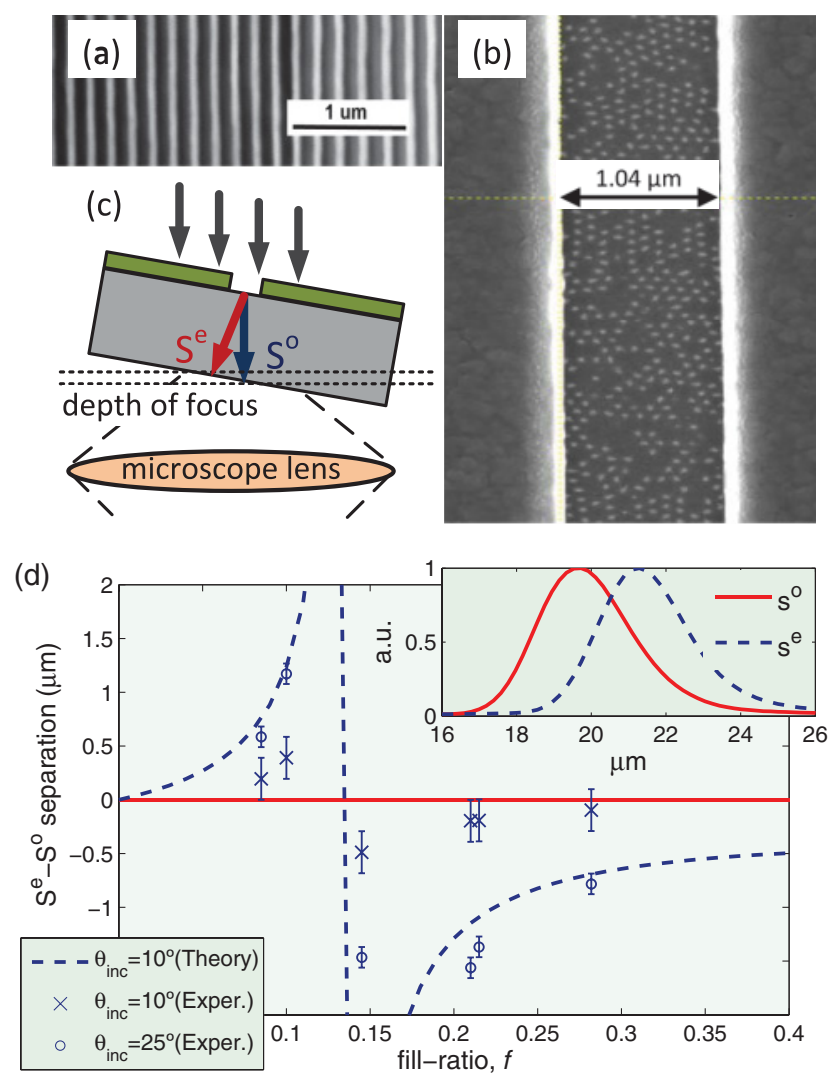

FIG. 2. (Color online) SEM images of a $\mathrm{Ag} / \mathrm{AAO}$ nanowire composite in (a) side view, and (b) top view of the slit region; (c) observation scheme; (d) birefringence separation $\left(S^{e}-S^{o}\right)$ of observations with different fill-ratios and incidence angles. Inset: $S^{e}$ and $S^{\circ}$ transmitted power flux from a sample of $\alpha(0.21, \sim 680 \mathrm{~nm})=$ -1.48 and $10^{\circ}$ incidence angle.

( $\sim 0.5 \mu \mathrm{m})$ was then $e$-beam evaporated on the top side, and a thin slit of $\sim 1 \times 100 \mu \mathrm{m}$ was opened using focused ion beam [Fig. 2(b)]. Finally, a circular hole of $\sim 0.5 \mathrm{~cm}$ diameter was opened at the bottom $\mathrm{Al}$ substrate, using a chemical etching process.

\section{EXPERIMENTAL RESULTS AND DISCUSSION}

\section{A. Birefringence swap}

The purpose of the thin slit was to allow a transmission beam entry narrow enough to resolve the birefringence separation at the exit side of the thin film [slit width set to $\sim(h / 2) \tan \left(\theta_{\text {inc }}\right) \simeq 1 \mu \mathrm{m}$, where $h$ is the sample thickness]. An optical transmission microscope was then used to observe the exiting birefringence with the samples placed in a tilted position and the slit parallel to the (horizontal) focal plane [Fig. 2(c)]. The transmitted image exhibited the slit in red color $(\lambda \sim 680 \mathrm{~nm})$, which is consistent with the increasing attenuation at shorter wavelengths indicated by the model results. Images of the separated exiting component were successively recorded by switching the polarizer next to the source. The images were treated [two-dimensional (2D) autoconvolution to eliminate noise and highlight the signal peak] and compared to determine the average separation distance. The inset in Fig. 2(d) illustrates the observed results from a sample with $\alpha(0.21,785 \mathrm{~nm})=-0.4$, using a $10^{\circ}$ incidence angle. A clear separation $\left(S^{e}-S^{o}\right)$ is seen between the maxima of the ordinary and extraordinary components. Figure 2(d) shows the results from several samples with different fill-ratios and incidence angles, including the model prediction curve for a $10^{\circ}$ incidence angle (dashed curve). The curve and results clearly show an increasing larger separation distance near $f \sim 0.15$, where the transition to HD takes place. This extreme separation results from the strong dispersion of the extraordinary component, and the change of sign indicates the swap with the ordinary component. Following the early described behavior, near the transition the $S^{e}$ vector gradually approaches the longitudinal direction, and when the elliptic dispersion changes into hyperbolic, it jumps to the opposite direction crossing with $k^{e}$ and $S^{o}$. Despite the differences between the predicted and experimental magnitudes, the observed results clearly show the swap between the birefringence components at the expected 0.15 fill-ratio. The differences are a most probable result of the spatial dispersion limitations of the model, which become significant at this region.

\section{B. Knife-edge refraction analysis}

To further test the extreme behavior of the extraordinary wave near the transition region, a second refraction study was performed at each side of the transition, using an extended analysis of the knife-edge technique. ${ }^{21,22}$ A single sample with $f=0.10$ and $20-\mu \mathrm{m}$ thickness was analyzed under monochromatic excitation in the near-infrared $(\lambda=1300 \mathrm{~nm})$ and visible light $(\lambda=785 \mathrm{~nm})$ regions. According to Fig. 1(a), such conditions correspond respectively, to a region of $\alpha<0$ and $0<\left(\alpha, n_{l}^{2}\right)<1$, as a result of an IP and near IP response. In these regions, one should detect three distinct refraction regimes [Fig. 3(a)]: a negative refraction (refraction as with

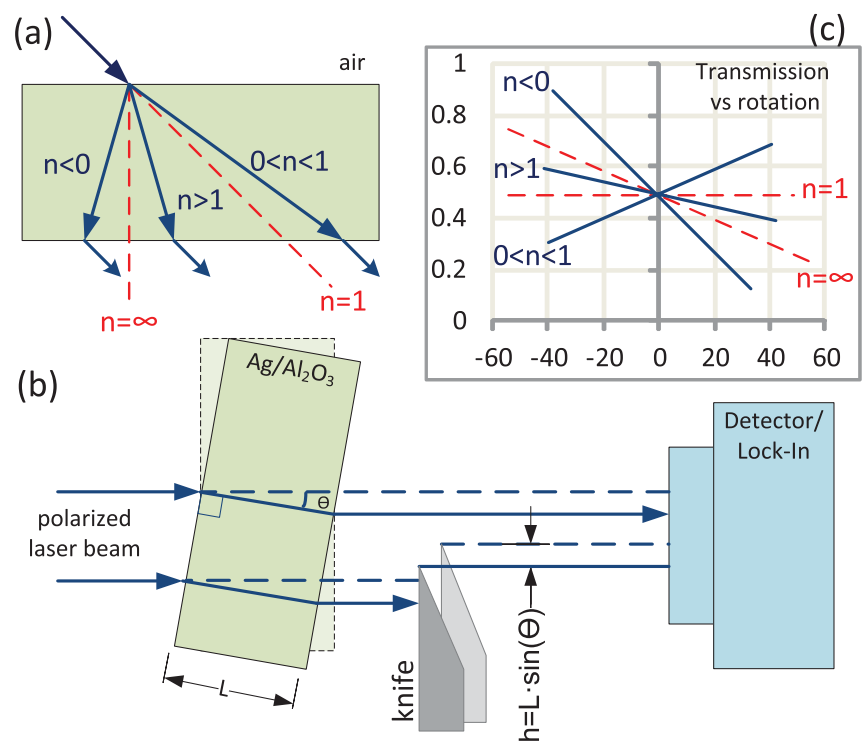

FIG. 3. (Color online) (a) Three distinct refraction regimes; (b) setup scheme illustrating an ideal refraction in the $n=\infty$ regime limit; (c) expected linear variation of the transmission with incidence angle, for each refraction regime. 
a refractive index of $n<0$ ) for $p$-polarization incidence with $\lambda=1300 \mathrm{~nm}$; a low refraction $(0<n<1)$ for $p$-polarization incidence with $\lambda=785 \mathrm{~nm}$; and a regular positive refraction $(n>0)$ for $s$ polarization with either $\lambda$. The sample was placed between the polarized laser source and a knife edge followed by the detector [Fig. 3(b)]. First, the knife edge was moved to the position where it blocked $\sim 50 \%$ of the transmitted power. Then, the sample was rotated around an axis orthogonal to the laser and the variation of the transmission was evaluated against the rotation angle. To reduce scattering effects, the measured power was normalized to that measured at the same angles without the knife edge. This procedure did not completely eliminate the scattering, and from Fig. 3(a) one can understand that this effect should become stronger for larger incidence angles in negative refraction conditions (larger deviation from the incidence direction). A Fabry-Pérot contribution was also found, ${ }^{23}$ adding a sinusoidal modulation to the expected linear variation of the transmitted power with rotation angle (for small variations, the transmitted power results proportional to the transmitted angle, which is proportional to the incidence angle). Fitting the experimental data, one can extract the slope $(\mathrm{m})$ of the linear contribution. From the setup scheme [Fig. 3(b)], one can understand that two slope values should mark the separation between distinct refraction regimes [Figs. 3(a) and 3(c)]. A $m=0$ slope, which would result from a $n=1$ refraction (no refraction), separates the low refraction regime from the regular positive refraction. ${ }^{22}$ The second separation is a slope of $m<0$ that divides the latter from the negative refraction regime. This slope can be determined from the dependence of the transmitted power on the knife-edge displacement and from geometric considerations of a refraction normal to the interface $\left(\theta_{t r}=0\right)$, as by an ideal isotropic medium with $n=\infty$ [Fig. 3(b)]. The dependence was measured using a piezoelectric stage for small displacements around the position of $\sim 50 \%$ transmitted power.

The $\lambda=785 \mathrm{~nm}$ experimental results are illustrated in Fig. 4(a). The thick dot and dashed lines represent, respectively, the $n=1$ and $\infty$ regime limits, and the thin dashed curves represent the fitting results of the transmitted power variation for $p$ - and $s$-polarization incidences. From the fitting procedures it results that for $s$ polarization, the slope value is negative, with magnitude below the $n=\infty$ mark (positive refraction), and for $p$ polarization, the slope is positive, indicating the theoretically expected low refraction. For $p$ polarization with $\lambda=1300 \mathrm{~nm}$, the experimental data clearly showed the effects of the mentioned strong scattering with increasing angle [inset of Fig. 4(b)]. To remove this behavior, a centered Gaussian fit was subtracted from the data. The result is illustrated in Fig. 4(b) together with the $s$-polarization results and the $n=\infty$ refraction mark. For $s$ polarization, the fitted slope is negative with magnitude below the $n=\infty$ mark (regular refraction), whereas for $p$ polarization is negative with magnitude well above the mark, indicating the negative refraction. As previously mentioned, the results of this experiment represent an extension of the work performed before by Hoffman et al. ${ }^{21}$ on epitaxially grown InGaAs/AlInAs nanolayered composites, using collected data from a large spectral range. Here, we used a nanocomposite with an equivalent optical response produced using a straightforward electrochemical method. This metamaterial was selectively studied on each

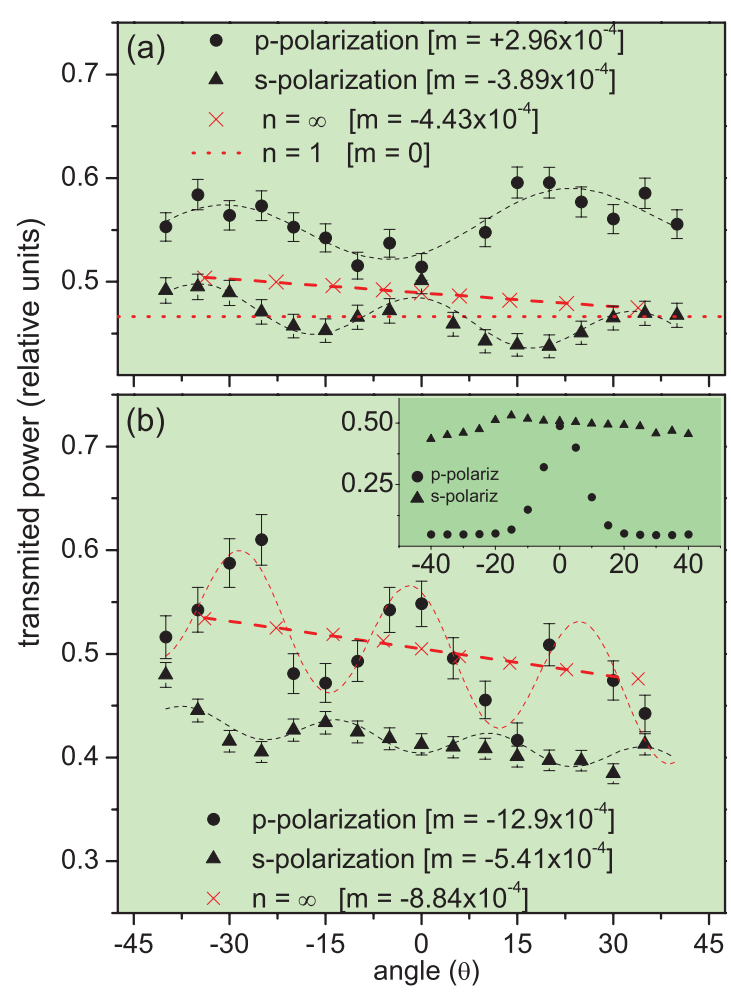

FIG. 4. (Color online) Knife-edge experimental results with (a) $\lambda=785 \mathrm{~nm}$ and (b) $\lambda=1300 \mathrm{~nm}$ radiation. Inset (b) shows the unfiltered data (strong scattering with $p$ polarization).

side of the transition, allowing a detailed confirmation of the full envisioned extreme dispersive behavior. This narrow-band approach was also used in order to emphasize the possibility of engineering almost any refraction angle within the metamaterial $\left(180^{\circ}\right.$ angle freedom). The present experiment also represents an extension of a recent work by Noginov et al. on this nanocomposite. ${ }^{22}$ Although the negative refraction regime was not studied, in such work the normal and low refraction slopes results ( $p$ and $s$ polarizations) of a sample studied under $\alpha(0.08, \sim 632 \mathrm{~nm})=0.38$ conditions are nearly one order of magnitude higher than those resulted here with a sample under close conditions $\alpha(0.10,785 \mathrm{~nm})=0.27$. From Fig. 3(b), we can qualitatively infer that this apparent inconsistency is due to the difference in the thickness of the samples.

\section{Model predictions and results of other works}

The $\alpha$-map modeling results were also used to test its consistency with a variety of optical phenomena identified in other studies performed on nanowire composites. In Table I, the $\alpha<0$ sample conditions studied in Ref. 24 are consistent with the demonstrated negative refraction, and the angular deviation closely follow the quantification solution described above. Furthermore, the $4.5-\mu \mathrm{m}$-thick sample studied under $\alpha(0.27, \sim 660 \mathrm{~nm})=-1.48$ conditions with an incident angle of $30^{\circ}$, produced an estimated birefringence separation of $\sim 1 \mu \mathrm{m}$. An approximate result was obtained here under similar conditions, with an incident angle of $25^{\circ}$ [Fig. 2(d)]. In Refs. 22 and 25, the identified transition to HD and minimal FWHM (transmittance peak full width at half maximum) both 
TABLE I. Identified phenomena in other studies performed on nanowire composites.

\begin{tabular}{llcrr}
\hline \hline & & \multicolumn{2}{c}{ Used conditions } \\
\cline { 3 - 5 } Reference & Identified phenomena & Composite & $f$ & $\lambda(\mu \mathrm{m})$ \\
\hline 24 & Negative refraction & Ag/AAO & 0.32 & 0.78 \\
22 & Transition to HD (IP) & Ag/AAO & $\sim 0.08$ & 0.84 \\
25 & Minimal FWHM & Ag/AAO & $\sim 0.08$ & 0.87 \\
26 & Transition to HD & Au/AAO & 0.15 & 0.4 \\
27 & Transition to HD & Au/AAO & 0.10 & 0.65 \\
8 & Hyperlensing & Au/AAO & 0.22 & 0.76 \\
\hline \hline
\end{tabular}

agree well with the $\alpha$-map result for the estimated 0.08 fillratio. Note that Eq. (4) in Ref. 25 indicates the transmittance profile dependence on the sample thickness, which directly affects the resulting $v$-shape curve aperture of the studied FWHM as a function of wavelength. The transition to HD studied in Refs. 26 and 27 refers to the limit of the observed extinction peak shifting (red peak in Ref. 27), which would result from a maximum incidence angle and high aspect ratio. In Fig. 1(a), one can see how this peak limit also shifts with the fill-ratio. At the transition to HD where we find a strong spatial dispersion, Ref. 26 has detected (after an annealing treatment) a splitting of the two transverse magnetic mode solutions of the Maxwell's equations. In Ref. 8, one has the most flattened equifrequency curve (just as in the represented $\alpha$-map for Ag/AAO), which is consistent with the canalization regime proposed by M. Silveirinha et al. ${ }^{28}$

\section{CONCLUSIONS}

To summarize, Ag/AAO nanowire composites were designed, fabricated, and used to experimentally demonstrate hyperbolic dispersion of visible light, in accordance with the Bruggeman model results. At the transition to hyperbolic dispersion, the normal surface analysis can still be use to describe the extreme behavior of the extraordinary component. The predicted swap between the extraordinary and ordinary components was demonstrated, and the negative refraction was detected as one among three distinct refraction regimes offered by this composite. The exceptional properties of this metamaterial make it attractive for a variety of remarkable applications, ranging from optical microscopy and cloaking devices to several plasmonic applications.

\section{ACKNOWLEDGMENTS}

This work was supported in part by the Project No. CERN/FP/116320/2010. Funding from FCT through the Associated Laboratory-IN is acknowledged. C.T.S. and J.M.T. are thankful for FCT Grants No. SFRH/BD/38290/2007 and No. SFRH/BPD/72329/2010. J.O.V. acknowledges financial support through FSE/POPH. J.P.A. also thanks Fundação C. Gulbenkian for financial support under the "Programa Gulbenkian de Estímulo a Investigação Científica."

\section{APPENDIX A}

For the sake of completeness, in this appendix we present the derivation of the Bruggeman formula. It results from the general expression of effective medium ${ }^{15}$

$$
\frac{\epsilon-\epsilon_{h}}{\epsilon-\eta \epsilon_{h}}=f_{a} \frac{\epsilon_{a}-\epsilon_{h}}{\epsilon_{a}-\eta \epsilon_{h}}+f_{b} \frac{\epsilon_{b}-\epsilon_{h}}{\epsilon_{b}-\eta \epsilon_{h}},
$$

where $\epsilon_{a}$ is the dielectric function of inclusions in a background with dielectric function $\epsilon_{b}, f$ and $\eta$ are the fill-ratio and screening factor associated with each phase, and $\epsilon_{h}$ is the dielectric function of a host medium. If $\epsilon_{h}$ equals 1 (void medium), we have the Lorentz-Lorenz expression and if it equals $\epsilon_{b}$, we have the Maxwell-Garnett expression. Note that if $f_{a}>f_{b}$, then a more appropriate choice in the MaxwellGarnett case is for $\epsilon_{h}$ to be equal to $\epsilon_{a}$. However, the resulting values of $\epsilon$ are different for the two choices. Bruggeman resolves this problem by proposing that neither phase should be given preference, but that the inclusions should be considered as being embedded in the effective medium itself. In the above formulation, this is equivalent to choosing $\epsilon_{h}=\epsilon$, in which case the left-hand side of the equation vanishes and we have

$$
0=f_{a} \frac{\epsilon_{a}-\epsilon}{\epsilon_{a}-\eta \epsilon}+f_{b} \frac{\epsilon_{b}-\epsilon}{\epsilon_{b}-\eta \epsilon} .
$$

This is the Bruggeman formulation, which is a quadratic equation with solutions given by Eq. (1). It is a shapedependent response, ${ }^{14}$ and in the present case, a longitudinal component arises with an infinitely large screening factor, which results due to the long aspect ratio of the nanowires.

An alternate $\alpha$-map was calculated using the MaxwellGarnet formulation. The comparison between this and the Bruggeman's $\alpha$-map in Fig. 1 indicates an almost perfect agreement of the HD transition curve, in all considered regions.

\section{APPENDIX B}

In this appendix, we present the derivation of the formulas used in the above normal surface analysis. The wave propagation in lossless uniaxial media satisfies the following known equation, which results from the Maxwell's theory:

$$
\left(\frac{k_{x}^{2}+k_{y}^{2}}{n_{e}^{2}}+\frac{k_{z}^{2}}{n_{o}^{2}}-\frac{\omega^{2}}{c^{2}}\right)\left(\frac{k^{2}}{n_{o}^{2}}-\frac{\omega^{2}}{c^{2}}\right)=0 .
$$

By substituting in Eq. (B1) $k_{x}=0, k_{y}^{2}=[(\omega / c) n]^{2}-k_{z}^{2}$, and $k_{z}=n(\omega / c) \cos (\theta),{ }^{29}$ we obtain the extraordinary wave equation [Eq. (2)] from the first factor and the ordinary wave equation from the second. It also follows from the Maxwell's 
equations that

$$
D \cdot E=\frac{D^{2}}{n^{2} \epsilon_{0}}=D E \cos (\delta)
$$

and

$$
\begin{aligned}
|D \times E| & =D^{2}\left(\frac{1}{n_{e}^{2}}-\frac{1}{n_{o}^{2}}\right) \sin (\delta) \cos (\delta) \\
& =D E \sin (\delta),
\end{aligned}
$$

where $\delta$ is the walkoff angle. By dividing these two equations, we obtain

$$
\tan (\delta)=\frac{n^{2}(\theta)}{2}\left(\frac{1}{n_{e}^{2}}-\frac{1}{n_{o}^{2}}\right) \sin (2 \theta),
$$

and, finally, by substituting the regular Snell law $n_{i n c} \sin \left(\theta_{i n c}\right)=n_{t r} \sin \left(\theta_{t r}\right)$ in Eqs. (2) and (B4), we then obtain Eq. (3). *jearaujo@fc.up.pt

${ }^{1}$ R. Shelby, D. R. Smith, and S. Schultz, Science 292, 77 (2001).

${ }^{2}$ V. G. Veselago, Sov. Phys.-Usp. 10, 509 (1968).

${ }^{3}$ J. B. Pendry, Phys. Rev. Lett. 85, 3966 (2000).

${ }^{4}$ D. R. Smith and D. Schurig, Phys. Rev. Lett. 90, 077405 (2003).

${ }^{5}$ D. R. Smith, P. Kolinko, and D. Schurig, J. Opt. Soc. Am. B 21, 1032 (2004).

${ }^{6}$ V. A. Podolskiy and E. E. Narimanov, Phys. Rev. B 71, 201101 (2005).

${ }^{7}$ Z. W. Liu, H. Lee, Y. Xiong, C. Sun, and X. Zhang, Science 315, 1686 (2007).

${ }^{8}$ B. F. Casse, W. T. Lu, Y. J. Huang, E. Gultepe, L. Menon, and S. Sridhar, Appl. Phys. Lett. 96, 023114 (2010).

${ }^{9}$ D. P. Gaillot, C. Croenne, F. Zhang, and D. Lippens, New J. Phys. 10, 115039 (2008).

${ }^{10}$ Z. Ma, P. Wang, Y. Cao, H. Tang, and H. Ming, Appl. Phys. B 84, 261 (2006).

${ }^{11}$ M. Moskovits, S. J. Lee, and A. R. Morrill, J. Am. Chem. Soc. 128, 2200 (2006).

${ }^{12}$ D. A. G. Bruggeman, Ann. Phys. (Berlin) 24, 636 (1935).

${ }^{13}$ C. T. Sousa, D. C. Leitao, M. P. Proenca, A. Apolinario, J. G. Correia, J. Ventura, and J. P. Araujo, Nanotechnology 22, 315602 (2011).

${ }^{14}$ W. S. Cai, U. K. Chettiar, A. V. Kildishev, and V. M. Shalaev, Nat. Photonics 1, 224 (2007).

${ }^{15}$ D. E. Aspnes, Am. J. Phys. 50, 704 (1982).

${ }^{16}$ W. T. Lu and S. Sridhar, Phys. Rev. B 77, 233101 (2008).
${ }^{17}$ J. Jackson, Classical Electrodynamics, 3rd ed. (Wiley, New York, 1998).

${ }^{18}$ H. Masuda and K. Fukuda, Science 268, 1466 (1995).

${ }^{19}$ K. Nielsch, J. Choi, K. Schwirn, R. B. Wehrspohn, and U. Gosele, Nano. Lett. 2, 677 (2002).

${ }^{20}$ G. Sauer, G. Brehm, S. Schneider, K. Nielsch, R. B. Wehrspohn, J. Choi, H. Hofmeister, and U. Gosele, J. Appl. Phys. 91, 3243 (2002).

${ }^{21}$ A. J. Hoffman, L. Alekseyev, S. S. Howard, K. J. Franz, D. Wasserman, V. A. Podolskiy, E. E. Narimanov, D. L. Sivco, and C. Gmachl, Nat. Mater. 6, 946 (2007).

${ }^{22}$ M. A. Noginov, Y. A. Barnakov, G. Zhu, T. Tumkur, H. Li, and E. E. Narimanov, Appl. Phys. Lett. 94, 151105 (2009).

${ }^{23}$ J. Kanungo and J. Schilling, Appl. Phys. Lett. 97, 021903 (2010).

${ }^{24}$ J. Yao, Z. W. Liu, Y. M. Liu, Y. Wang, C. Sun, G. Bartal, A. M. Stacy, and X. Zhang, Science 321, 930 (2008).

${ }^{25}$ M. A. Noginov, L. V. Alekseyev, E. E. Narimanov, T. Tumkur, H. Li, and Y. A. Barnakov, Appl. Phys. Lett. 97, 131107 (2010).

${ }^{26}$ R. J. Pollard, A. Murphy, W. R. Hendren, P. R. Evans, R. Atkinson, G. A. Wurtz, A. V. Zayats, and V. A. Podolskiy, Phys. Rev. Lett. 102, 127405 (2009).

${ }^{27}$ R. Atkinson, W. R. Hendren, G. A. Wurtz, W. Dickson, A. V. Zayats, P. Evans, and R. J. Pollard, Phys. Rev. B 73, 235402 (2006).

${ }^{28}$ M. G. Silveirinha, P. A. Belov, and C. R. Simovski, Phys. Rev. B 75, 035108 (2007).

${ }^{29} \mathrm{~A}$. Yariv and P. Yeh, Optical Waves in Crystals: Propagation and Control of Laser Radiation (Wiley, New York, 2002). 